\title{
Exploration of Pipelines from the use of GPR Data by Neural Network
}

\author{
Kode Rajiv, Booba Basaveswara Rao, G Ramesh Chandra, N V Ganapathi Raju
}

\begin{abstract}
Noticing about the buried pipes is a important issue, In many regions of the world. In spite of the fact that several techniques are there. This literature is used to find out the underground pipes automatically that provides accuracy execution is underway. Which gave amazing results Achieved by the deep learning of the different discoveries found in this article offer a pipeline to detect anti-personnel pipes Adaptive Neural Networks ( applied to the Ground Penetrating Radar (GPR). The proposed algorithm is suitable to recognize if the scanning format has been received. The acquisition of GPR has a track of anti-personnel pipes. The validity of the said system is made on a real GPR receipt, although systematic training can be done to have relied upon data generated by achievements. Based on the results $95 \%$ of the accuracy of detection got achieved without testing acquisition of pipes.
\end{abstract}

Index Terms: GPR, Pipes, CNN, Data Acquisition, B-Scan Image, AUC, ROC.

\section{INTRODUCTION}

Pipes and inflammable remnants of war are contagious region in over 90 countries around the world Serious and continuing threats to citizens [1]. That Figure. 1 of the victims of pipes are not well known It is evaluated that about 26,000 people per year Killed or forbidden by the pipes. So the evolution of Methods of pipes production for mining the site is extremely important. The problem of solving the mining is in two separate steps: (i) Find the buried object and (ii) separation of objects. In particular, find the objects which are there in buried it is a threat. The distribution of objects is a process From Discrimination of Interested Objects (e.g., Pipes) from other buried purposes (eg, Flooding) [2].

This article focuses at the first step. In fact, disclosing pipes is a challenge because it consists of various factors: large Diversity of pipe rales, soil conditions, soil conditions, climate Conditions, the availability of human and natural waste to be named two or three. Traditional methods are electromagnetic (EMI) intended specifically for detection Metal targets. However, many pipes have been created Plastics and little or no metal. In this context, the earth the integrated radar system (GPR) seems to be appropriate [3], [4], [5]. In fact, GPR sensors work by measuring electromagnetic reflection Pulse of suspension in dielectric power Real Estate So You Can Find Non - Metal Purposes by

Their electric colleagues with the soil environment. However, GPR sensitivity to water change is also available some shortcomings. In fact, GPRs tend to be alert Land and soil. Here the underground objects are detected by GPR data.
In many deals, GPR and image Processing techniques are required automatically Find Hidden Objects. In principle, these methods are the first Perform step-by-step steps to process data running data tasks Normal, correction for variations in the distance from the top and speed, removing the station's impact due to a systematic response, Background shading [6], [7], [8]. The data is then processed Scan to find the presence of buried goals. This Purpose, both approach and functions Techniques are available. Pattern model target approach to individual hyperbole in GPR images Make changes [9] Hough or technical correctness [10].

However, the variability of the GPR system to regional changes Environmental conditions lead to many different answers From the funeral object that blocks the proper detection of hyperbole. In this scenario, the algorithm is based on statistics Download features from GPR images, including histograms Description [11], Gradient Histogram Oriented [12], Hidden the Markov model [13], by the way, proved to be strong Application to a wide variety of data. In this article, we offer a detection algorithm for anti-personnel pipes Relay Network Study (CNN) [14] To analyze GPR B-scan (for example, 2D portrait image Underground Pie). Our approach belongs to functionality Technical specifications but inverts sample samples that are commonly used. Of course, we use the data-based approach Learn a live bumper feature GPR image rather than modelling or manually The special formula formulated in [15] to identify anti-personnel pipes. Get in particular, we focus on the necessary pipeline Previous processing images (e.g., Only sync and Removing a direct antenna).

The advantage of use alternative approaches to other modern solutions are call attention by our experimental campaigns running on GPR Actual data obtained from the test area. In particular:

(i) Our algorithms do not rely on any sample analysis Easy error due to simple assumptions or samples Simplicity (eg Linearization, etc.).

(ii) a request Methods can work on a small patch with high images precision, clear a path for the specific localization of the target.

(iii) $\mathrm{CNN}$ requested only training for audio-targeted signatures learn a good way to download Actual GPR data.

(iv) The build ability is also true Buying in training steps can improve the system Reaching 95\% accuracy.

Revised Version Manuscript Received on 16 September, 2019.

Kode Rajiv, Assoc. Professor in information Technology, GRIET Hyderabad

Booba Basaveswara Rao, Computer Center, ANU-Guntur

G Ramesh chandra, PhD from JNTUH from CSE Dept in the area of image processing.

N V Ganapathi Raju, Department of I.T., at GRIET, Hyderabad 


\section{LITERATURE REVIEW}

\section{A. GPR Data Acquisition:}

For a given location, a GPR transmitters transmit electromagnetic pulses Soil and antenna are measured the signal size is a function of time. This lonely form Recorded by GPR with antenna at constant the location is called A-scan. The structure of A-scan It is strongly influenced by the environment Radiation emissions. If the media has an existing domain. The different electronic static A-scan will be complicated Reflection in the regional interface. When moving GPR An antenna on the line can merge A-scan Create a two-dimensional data set called B-scan. In short, a B Scan Image representing the vertical part of the soil in the pixel intensity is the amount of the receipt Signal. So scanning b provides a more efficient way for visibility and essential environmental characteristics. You can usually see two different patterns in Scan B:

(i) Hippolyte obtained by reflection Electromagnetic signs on small cells.

(ii) The soil layers will have different impedance because of that linear segments occurs.

\section{B. Revolutionary neural network:}

Advanced Nervous Network (CNN) are complex calculations Of the largest number of connected nodes Numeric parameters can be flexible to learn complex and Nonlinear functions [14], [16]. The network node is stacked the multilayer layer performs a simple operation, CNN layers typically have: Conclusion: The transition layer is a filter line $H$. Given the " $x$ " character, enter the result from each filter the right part of linear change. Max Match: This layer suggests input $\mathrm{x}$ from Drag a short window and reduce it the value for each window position. ReLU: Rectified Linear Device (ReLU) Applies Adjustments the maximum function $(0 \mathrm{x})$ to the $\mathrm{x}$ input port, thus shortening Negative values to zero [17].Internal Product: Linear Combined Technology All samples from input $\mathrm{x}$. SoftMax: normalizes input values in the range $[0 ; 1]$ and make sure they are full. This is special Useful at the end of the network to explain the result is the probability value. By providing $\mathrm{CNN}$ with a group of labeled data (e.g., Images Belonging to different known species) and to be minimized Cost Functions at output of CNN Ultimate Layer (For example, filter value in the layer, translation, etc.)

It is allowed so that CNN learns how to quote automatically Separate data features (e.g., Image type). In the imagery scenario, $1^{\text {st }}$ layer of the network in general, learn base concepts such as edges and robustly layers, deep layers, find complex images Model. Finally, bottom layer has a dataset Combined with the pay function It is reduced. For example, in the context of the binary image Classification, the final layer consists of 2 nodes (e.g. For Class), which limits distribution, probably over Visible types. That the given node value be owned to the last layer displays the availability of the input image for the visible class. For a particular imaging task we need a trained CNN model: (i) Establish meta-parameters of $\mathrm{CNN}$, e.g., that the order is applied to a number of layers and shape of filter in the convolution layers. Deter pipes the appropriate function to calculate the cost of the period Training Process (iii) Prepare a database (possibly larger) from workout and image testing, annotated with some transactions (eg GPR B-scan in our work). (III) Distribution system, the purpose is to understand Scanning obtained through the purchase of GPR is traceable Buried pipes detection objects.

This officially means Acceptance, as my picture implies b analysis and results Single label Indicates possible absences (e.g. $=0)$ or presence $($ Eg., Ltd. = 1) of the object. The reason for the proposed technique is that of B scans the current hyperbolic current exists in GPRs Analyze structure of the funeral object as shown in Part II. Conversely, if the soil is free, $b$ - scans do not show the hyperlink. Now it will allow to use the CNN-based image recognition system discrimination between the scan scans with these traces. The detected detection system pipeline is displayed First, CNN is being trained to discriminate Patch tracking objects (e.g., Hyperbole) or not (e.g. Background). When the system is aim is to notice whether an object is buried or a b-scan is taken to separate Patch. Each gap is to be tested against the CNN. Elections it is linked from $\mathrm{CNN}$ to each of the gap final results. A complete explanation is ahead of us for every step.

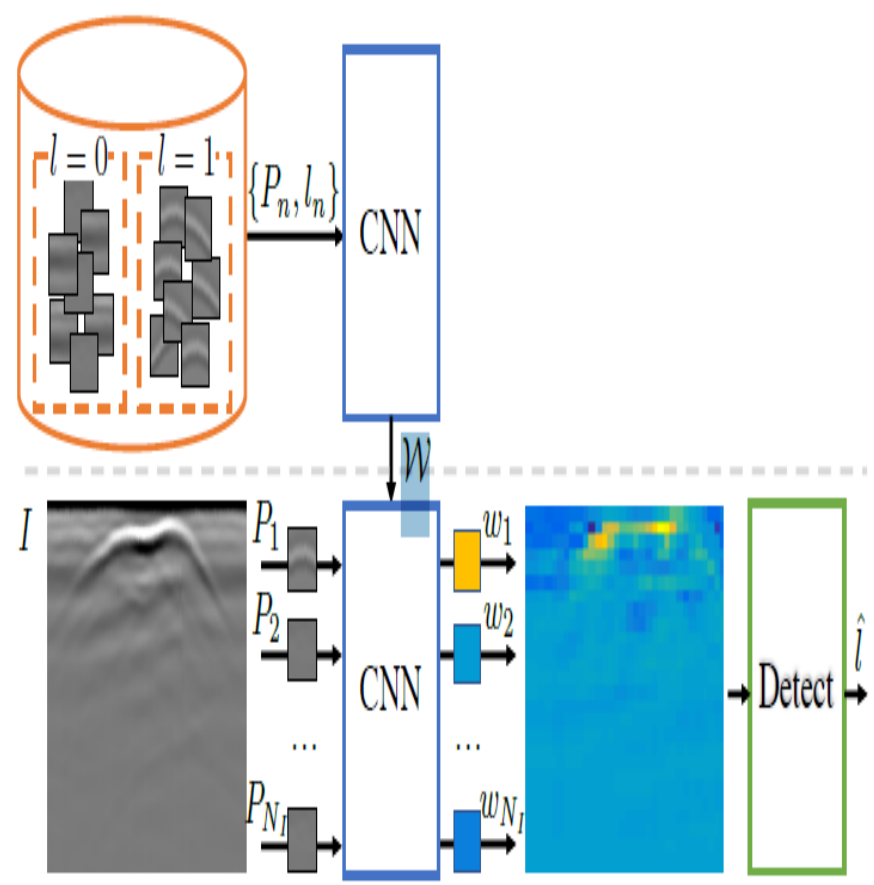

Fig 1: Open pipe network. The learning process at the top of the system Deploy the bottom.

System training provided by CNN N technology we need In order to deter pipes its set of weights W (eg Filter coefficients, internal weight.) For specific tasks. It is CNN training was conducted as in standard two-level observation Questions. Do we use the B database? Size of Part B PN; n2 [1; Ntrain is divided into two types, ie. Objects Compared to the background (see Figure 1). 
Each patch is attached the ln tag depends on its type: Pn fragment of the background There is background noise labelled $\ln =0$; the object Pn object with part of the hyperbolic is indicated with $\ln =1$. CNN is supplied with all available paints FPN; LNG; n2 [1; Ntrain and learn related tags Patch. When CNN is trained, it can be used to classify (e.g. Label Attachment) New patches have never been used in the training Step.

It is especially possible to power CNN with pn has no Pn tag and gets disproportionate vote Pn's capabilities belong to class objects. The high wn, Probably Pn contains hypothetical. System naming to find out if B-scan Image I was following the original stuff, I split it into NI Pn overlay patch; $\mathrm{n} 2[1 ; \mathrm{NI}]$ of size $\mathrm{B}$ ? b Pn patch is given to $\mathrm{CNN}$, which connects one voice to everyone One of them. The design is to delete the patch from the section My origin is related to only low pay On the contrary, the patch contains some of the hyperbola Applies to the high value of wn. An example is given Figure 1 shows the hyperbolic patch Places are hung from $\mathrm{CNN}$ to high voices (for example, yellow In the picture). After all my patches were rated, we found the presence from object to vein value. We officially got in touch Single label Show background $(\wedge 1=0)$ or objects (and so on). $=1)$ p Rules below:

$$
\hat{l}=\left\{\begin{array}{ll}
0, & \text { if } \max _{n}\left(w_{n}\right)<\Gamma \\
1, & \text { otherwise }
\end{array},\right.
$$

Where $\operatorname{maxn}(\mathrm{wn})$ extracts the maximum value among all wn; $\mathrm{n} 2$ [1;NI ], and $\Gamma$ is a threshold will be finalized upon a small training set of images. In this section, we provide detailed review information Network architects, data arrays, and test methods. Network Architects to Check Accessibility Using CNN to detect a buried pipes we've checked proposed projects using different network architectures. The architecture of the N1 is inspired by the popularity of LeNet [16], These are composed of twenty-kernel 2 cohorts Size 5? 5 ReLU and 2? 2 Maximum compliance, Accompanied by two fully connected layers of 500 and 2 cores, accordingly. The N2 architecture is a smaller version of the N1 Which ginger converter is minimized to 3 ? 3 and the number of nerve cells of the first fully bound layer Reduced to 250 .

Finally, N3 is version N1 with One layer of conversion is more than two. Theoretically, all test networks accept a patch for inclusion Small size B? We actually tested the patch for B $2 \mathrm{f} 32 ; 64 ; 128$ grams corresponding to 8, 16 and Correspondingly 32 centimetres. There are training courses using a stochastic gradient with a learning speed of 0: 1 the package of 64 download patches disables hacking about the assortment. The selected template is selected

As a minimum deduction for a compact validity in the first 10 epochs (eg complete pass through all coaching images). Training is done on the required GPU GeForce GTX 980 Less than one minute at a time. Test a data set to fully verify the suggested system, We strongly believe that the actual GPR data should be It is believed to be. For this cause the suggested pipeline Try only real data. In particular, the actual data used in these Workplaces are collected using existing GPR tools Radar IDS Aladdin (IDS Georadar srl) is a protected area DP polypropylene antenna $(9 \mathrm{~cm})$ with center frequency $2 \mathrm{GHz}$ speed. One pad, PSG [18], was Place between radar and ground tools to make sure exact measurement and constant orientation of the antenna by Trace to track.
In our preparation, here 9 various aims be entitled the nuances In the tomb were buried patterns of rubble and destruction Deterpipesd by very low clay content and rough texture, at a depth of about 10 centimeters. Then we checked the area so each scan corresponds to a time window of $20 \mathrm{~ns}$ and samples 384 times. We have a 114-bit scan $180 \mathrm{~cm}$ Read sample and curve 0: $4 \mathrm{~cm}$ Sample 0: $8 \mathrm{~cm}$ showing a piece of each of us Hand marked B - Scanning that have traces or not objects. This work applies only to B-scans automatically Resize the pixel / $\mathrm{cm}$ ratio used by $\mathrm{CNN}$ and Exceptions to 1st a very small number of rows of images with direct paths. From sender to recipient. An example of a formal view of the request form the system is a training strategy. Of course Effective training on the CNN network, thousands or even thousands of data sets
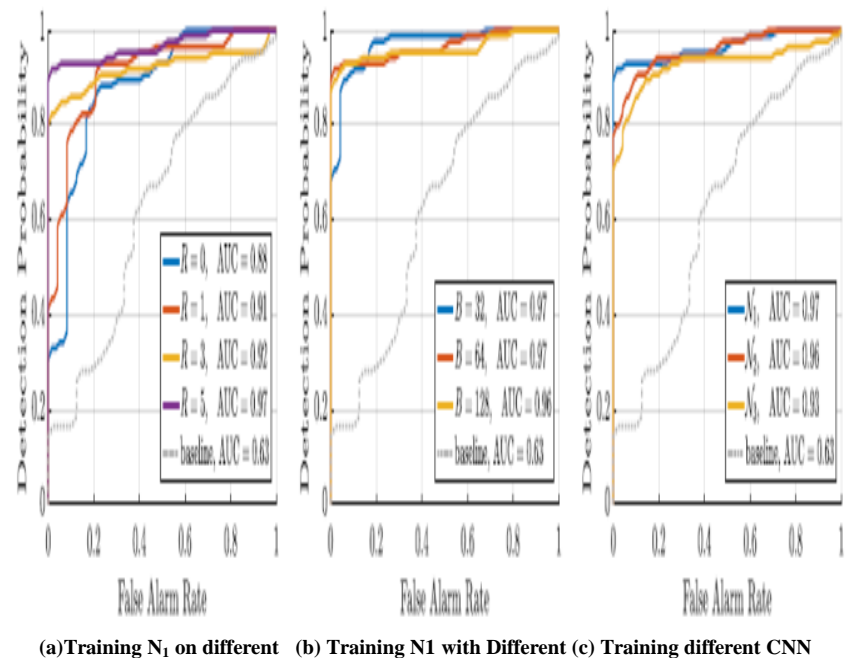

Training $\mathrm{N}_{1}$ on different (b) Training N1 with Different (c) Training different CNN datasets $D_{R}$ path size $B \quad$ architectures

Figure. 2: ROC curves obtained with the proposed solutions: (a) network $\mathrm{N} 1$ for different numbers $\mathrm{R}$ of training B-scans from real acquisitions; (b) network $\mathrm{N} 1$ for different patch sizes B; (c) different network configurations N1, N2 and N3, fixing $\mathrm{B}=64$ and $\mathrm{R}=5$.

\section{RELATED WORK}

Typically the image is used [14]. It is a big problem about the proposed pipeline, like a large number of tags Scanning by GPR B may not be easy. However, the solid idea of the suggested architecture is the possibility they are trained on images created by the still existing sound They can work when deployed to get a real GPR. To test this feature, we've created four different training courses Data set DR, R 2 f0; 1 ; $3 ; 5 \mathrm{~g}$ shows the number from actual scans of data used for training. In particular, D0 has only synthesized patches created by GPR Max simulation Program [19]. We created a 50: 000 background patch 50: 000 patches with split hip-hop fossils B - Scanning of different soil components (i.e. different Sand, clay and composition) or not Of various shapes (e.g., Slides and Cylinders) Constantly mixed intensity. Starting from D0, then we created D1, D3 and D5 by adding to D0 is only a gap of 1,3 or 5 scans - scans from actual purchases. 
It is main to observe Networks never use a real-time data entry patch Training. So here we assume that there is no information. The goals are available besides synthesis Create an image.

\section{RESULTS}

In this section we present the rating used and achieved results. The suggested metric rating depends on this Level 1, we appreciated our approach by getting it Work Curve (ROC). These curves constitute the availability of observation and false alarms for identical values. The probability is the percentage of B-scans exact objects found. Not true

The degree of notification is the availability of identifying object detected by a scanner that does not contain it. Good sensors are set by ROC under the curve (AUC) Predictive 1. Unintentionally forecasting is distinguished by a flat AUC 0: 5. As an extra meter, we also offer the accuracy of the discovery for the best choice. Measure the struggling about task they are considering

And make a easy basic solution inspired by The pre-screening method used in [12]. In particular, we calculated the average scan A for each b-scan and took it to the maximum

The High Energy Value returns to GPR. According to at this price level, we detected the existence of an object. All outputs are measured to the baseline.

The first result we confirmed our ability. This system is trained on synthetic data, but it also shows It is feasible to improve system performance by using Some up-to-date data in the training kit.

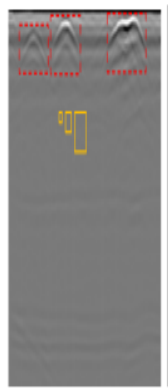

(a) B:xen

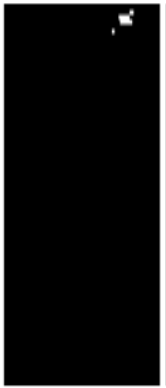

(b) D

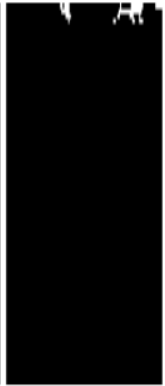

(i)

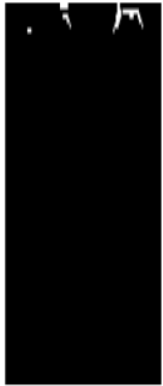

$\left(d D_{3}\right.$

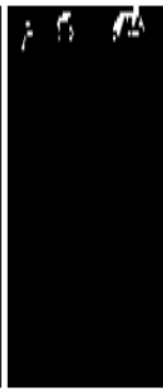

(b)
Fig 3: Examples of identifying masks noticed thresholding weights $w n$.

White areas indicate patches identifying as containing hyperbolic traces. (a) reference B-scan with three targets (dashed rectangles) and example of patches for B 2 f32; 64; $128 \mathrm{~g}$; (b) mask obtained for $\mathrm{R}=0$; (c) mask obtained for $\mathrm{R}=$ 1; (d) mask obtained for $\mathrm{R}=3$; (e) mask obtained for $\mathrm{R}=5$. this purpose, Figure 2 shows the ROC curves obtained using N1 trainings Copying with B $=64$ from D0, D1, D3 and D5, respectively. From this figure and the result as the number shown in Table I It is feasible to identify that when using synthesis data for training (eg D0), the distribution pipeline still finds buried objects with $83 \%$ accuracy. Additionally, by adding 1 to 5 backgrounds B - Real Data Scanning in Training Kit, Accuracy of observing Up to $95 \%$. Kindly notice that anyhow of the data.

\section{COMPARISON}

The used CNN has never been trained using real-life data hyperbolic trace. Basic scanning is only available $62 \%$ accuracy of the object. Fig 3 gives a good clarity about the role of use Different openings for pipes.

Especially given The B-Scans report has 3 purposes (Figure 3 (a)) using D0 to train only one goal detection system. With an escalator the number of exact background corrections that are visible during the training (Figure 3 (b-e)) the system recognizes all targets. After verifying the correctness of the training strategy, we tested The effect of using the input patch differs in size B. Figure $2 \mathrm{a}$ Shows the results obtained with the trained N1 architecture of D5 For B $2 \mathrm{f32}$; 64; 128g It is possible to note the results for $\mathrm{B}=32$ and $\mathrm{B}=64$ have little difference. On the other hand, By increasing the patch size to $\mathrm{B}=128$ system experience Slight decline in productivity. Of course, big patches are big Parts of the hyperbole, so CNN is not so generic it reaches the hyperbola of forms a little variation. We evaluate effect of using different networks Fig $2 \mathrm{c}$ displays ROC curve acquired using N1, N2 and N3 repair $\mathrm{B}=64$ and use D5 training data[19].

In fact, by reducing the size of CNN (eg. N2 and N3) less accuracy is observed. Table I displays the results of a certain number of accuracy and AUC for each test shown.

TABLE I: Numerical results for different proposed strategies

\begin{tabular}{ccccc}
\hline Algorithm & Patch Size $(B)$ & Dataset $(R)$ & Accuracy & AUC \\
\hline baseline & - & - & 0.62 & 0.63 \\
\hline $\mathcal{N}_{1}$ & 64 & 0 & 0.83 & 0.88 \\
& 64 & 1 & 0.85 & 0.90 \\
& 64 & 3 & 0.90 & 0.92 \\
& 64 & 5 & $\mathbf{0 . 9 5}$ & $\mathbf{0 . 9 7}$ \\
& 32 & 5 & 0.92 & $\mathbf{0 . 9 7}$ \\
& 128 & 5 & 0.94 & 0.96 \\
\hline $\mathcal{N}_{2}$ & 64 & 5 & 0.90 & 0.96 \\
\hline $\mathcal{N}_{3}$ & 64 & 5 & 0.88 & 0.93 \\
\hline & & & &
\end{tabular}

\section{Conclusion}

In this article, we discussed about a mining pipeline based on the B-scan image which are collected from GPR and got analized. The request the methods were depended completely for the use of vascular networks and it's completely automated. Valid on actual purchases of GPR indicates that the system gives up to $95 \%$ correctness. The need for small images to be processed in advance. The experimental results are optimistic that $\mathrm{CNN}$ was depended on pure synthesis data. However, by appending some GPR purchases tanks of the training image can dramatically increase Accuracy of detection. However, this system is not required they are trained on photos that describe specific objects of interest from actual purchases. For Pipes Scenarios Our approach is having a good importance possible In order to obtain some of the uncontrolled field pipes, and unpack the system to find objects that have never been earlier. Despite the successful presentation proposed the pipeline does not take full advantage of thinking GPR system. 


\section{FUTURE SCOPE}

In order to improve the future, future work will be Attention was paid to studying the effect of using various antennas polarization. The possibility Work directly in three-dimensional domains, not simply using a B Scan. Finally, we will apply the GPR in more detail Data search campaigns to study system generalization capability to different kinds of targets.

\section{REFERENCES}

[1] International Campaign to Ban Landmines, "Landmine monitor 2015," Human Rights Watch, 2015.

[2] T. R. Witten, "Present state of the art in ground-penetrating radars formine detection," in SPIE Detection and Remediation Technologies for Mines and Minelike Targets, 1998.

[3] L. P. Peters, J. J. Daniels, and J. D. Young, "Ground penetrating radaras a subsurface environmental sensing tool," Proceedings of the IEEE, vol 82, no. 12, pp. 1802-1822, 1994.

[4] Y. Liao, L. W. Nolte, and L. M. Collins, "Decision fusion of groundpenetratingradar and metal detector algorithms mdash; a robust approach,'IEEE Transactions on Geoscience and Remote Sensing, vol. 45, no. 2, pp. 398-409, 2007.

[5] D. J. Daniels, "GPR for buried landmine andied detection," in Unexploded Ordnance Detection and Mitigation. Springer, 2009.

[6] U. S. Khan and W. Al-Nuaimy, "Background removal from gpr data using eigenvalues," in Internarional Conference on Ground Penetrating Radar, 2010

[7] D. Carevic, "Clutter reduction and detection of minelike objects in ground penetrating radar data IJRTE using wavelets," Subsurface Sensing Technologies and Applications, vol. 1, no. 1, pp. 101-118, 2000 .

[8] A. H. Gunatilaka and B. A. Baertlein, "Subspace decomposition technique to improve gpr imaging of antipersonnel mines," in SPIE Detection and Remediation Technologies for Mines and Minelike Targets, 2000.

[9] G. Borgioli, L. Capineri, P. Falorni, S. Matucci, and C. G. Windsor,"The detection of buried pipes from time-of-flight radar data," IEEE Transactions on Geoscience and Remote Sensing, vol. 46, no. 8, pp. 2254-2266, 2008.

[10] H. Chen and A. G. Cohn, "Probabilistic robust hyperbola mixture model for interpreting ground penetrating radar data," in International Joint Conference on Neural Networks, 2010.

[11] H. Frigui and P. Gader, "Detection and discrimination of land mines in ground-penetrating radar based on edge histogram descriptors and a possibilistic $\mathrm{k}$-nearest neighbor classifier," IEEE Transactions on Fuzzy Systems, vol. 17, no. 1, pp. 185-199, 2009.

[12] P. A. Torrione, K. D. Morton, R. Sakaguchi, and L. M. Collins, "Histograms of oriented gradients for landmine detection in groundpenetrating radar data," IEEE Transactions on Geoscience and Remote Sensing, vol. 52, no. 3, pp. 1539-1550, 2014.

[13] P. D. Gader, M. Mystkowski, and Y. Zhao, "Landmine detection with ground penetrating radar using hidden markov models," IEEE Transactions on Geoscience and Remote Sensing, vol. 39, no. 6, pp. 1231-1244, 2001

[14] Y. Bengio, "Learning Deep Architectures for AI," Foundations and Trends in Machine Learning, vol. 2, no. 1, pp. 1-127, January 2009.

[15] L. E. Besaw and P. J. Stimac, "Deep convolutional neural networks for classifying gpr b-scans," in SPIE Detection and Sensing of Mines, Explosive Objects, and Obscured Targets, 2015.

[16] Y. LeCun, L. Bottou, Y. Bengio, and P. Haffner, "Gradient-based learning applied to document recognition," Proceedings of the IEEE, vol. 86,no. 11, pp. 2278-2324, 1998.

[17] V. Nair and G. Hinton, "Rectified linear units improve restricted boltzmann machines," International Conference on Machine Learning, 2010.

[18] M. Lualdi, "True 3d acquisition using gpr over small areas: A cost effective solution," in Symposium on the Application of Geophysics to Engineering and Environmental Problems, 2011.

[19] C. Warren, A. Giannopoulos, and I. Giannakis, "gprmax: Open sourcesoftware to simulate electromagnetic wave propagation for groundpenetrating radar," Computer Physics Communications, vol. 209, pp. 163 - 170, 2016.2017 (EUSIPCO)ISBN.

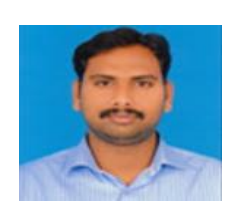

\section{AUTHORS PROFILE}

Dr. K. Rajiv, Ph.D from ANU-Guntur, M.Tech in SRKR Engg College from the (CSE), Bhimavaram. Presently working as Assoc. Professor in information Technology, GRIET Hyderabad. He published 12 articles in different Journals and Conferences. Till now he has 9years of Teaching and Research Experience in several organizations.

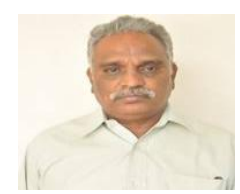

Dr. Bobba Basaveswara Rao Ph.D in ANU-Guntur. His areas of interest is Computer Networks, Network Security. He is working as Web Administrator in Computer Center, ANU-Guntur. He published 20 articles in various journals. He has 30 years of both Research and Teaching Experience.

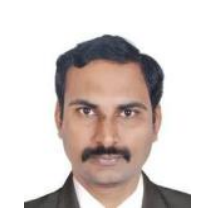

Dr. G. Ramesh Chandra got $\mathrm{PhD}$ from JNTUH from CSE Dept in the area of image processing. He is having 19 Years of experience in research and teaching. From 2013 he is in the role of Head of RCC and involved in both research and several RCC project works. He published 32 articles in National and International Journals and Conferences.

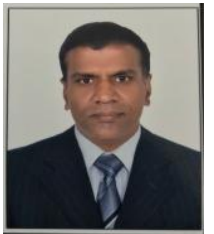

Dr. N. V. Ganapathi Raju, Department of I.T., at GRIET, Hyderabad. He is in the role of Associate Dean Alumni Affairs. Ph.D. from JNTUK, . M.Tech (C.S.T.) from AU-Vizag. He has total 18 years teaching and 7 years research experience. His research interests include Information Retrieval and Natural Language Processing, Data Science and Machine Learning. He published research articles in various International journals. He is Webmaster for GRIET Website. 